\title{
2.4 THE RELATION OF THE LOW FREQUENCY SOURCE TO THE CRAB PULSAR
}

\author{
KENNETH R. LANG \\ Cornell-Sydney University Astronomy Center, Arecibo Observatory, \\ Cornell University, Ithaca N.Y., U.S.A.*
}

\begin{abstract}
The view that the compact low frequency source and the pulsar NP 0532 are the same object is substantiated by an examination of the general properties of interstellar scattering. This scattering accounts for the observed angular size of the compact source, the observed pulse broadening of NP 0532, the continuum nature of the compact source, and the observed spectrum of both the pulsar and the compact source.
\end{abstract}

\section{Introduction}

Observations using an interferometer at $38 \mathrm{MHz}$ (Hewish and Okoye, 1964) and the lunar occultation technique at $26 \mathrm{MHz}$ (Andrew et al., 1964) first showed that the Crab Nebula contains a compact continuum source. Interplanetary scintillations of the source (Hewish and Okoye, 1965 and Bell and Hewish, 1967) indicated that it was unusually small $\left(\approx 0.1^{\prime \prime}\right.$ or $\left.10^{-3} \mathrm{pc}\right)$ and had a very high brightness temperature $\left(\approx 10^{14} \mathrm{~K}\right)$. Gower $(1967)$ found that the position of the compact source coincides with the south-preceding central star of the nebula, within the positional accuracy of $\pm 12^{\prime \prime}$ in $\alpha$ and $\pm 1^{\prime}$ in $\delta$. This central star was thought to be the neutron star remnant of the supernova explosion. The theoretical models which were thought to explain pulsars incorporated an object whose properties - small size, high brightness temperature, and association with a neutron star - are very similar to those of the compact source. Noting this similarity, Woltjer (1968) suggested that the compact source might be found to be a pulsar. When the pulsar NP 0532 was subsequently discovered and found to coincide with the central star of the supernova, there was naturally considerable speculation that the pulsar and the compact source were the same object.

\section{Angular Scattering by the Interstellar Medium}

One of the major obstacles to the view that the compact source is the pulsar NP 0532 is that the compact source is considerably larger than the pulsar. Angular scattering in the interstellar medium might, however, cause the pulsar to appear to be larger at lower frequencies. As long as the pulsar was much smaller than the rms scattering angle, $\theta_{\text {scat }}$, it would appear to have the size $\theta_{\text {scat }}$. The presence of such scattering has been inferred from the observed fluctuations in pulsar radiation intensity (Rickett, 1969; Lang, 1969). For example, if the scattering takes place midway between the pulsar and the Earth, the path difference between the direct and scattered rays from a pulsar at a distance, $D$, will be $D \theta_{\text {scat }}^{2} / 4$. Consequently, when pulsar signals are

* The Arecibo Observatory is operated by Cornell University under contract to the National Science Foundation and with partial support from the Advanced Research Projects Agency. 
observed over bandwidths larger than the decorrelation frequency

$$
f_{v}=4 c / D \theta_{\text {scat }}^{2},
$$

the scintillation patterns at different observing frequencies will interfere, and the observed intensity fluctuations will be considerably reduced.

Rickett (1969) and Lang (1971) have measured decorrelation frequencies for many nearby pulsars (Figure 1). These data indicate that

$$
f_{v} \approx 5 \times 10^{-9} v^{4} /\left(\int n_{e} \mathrm{~d} l\right)^{2},
$$

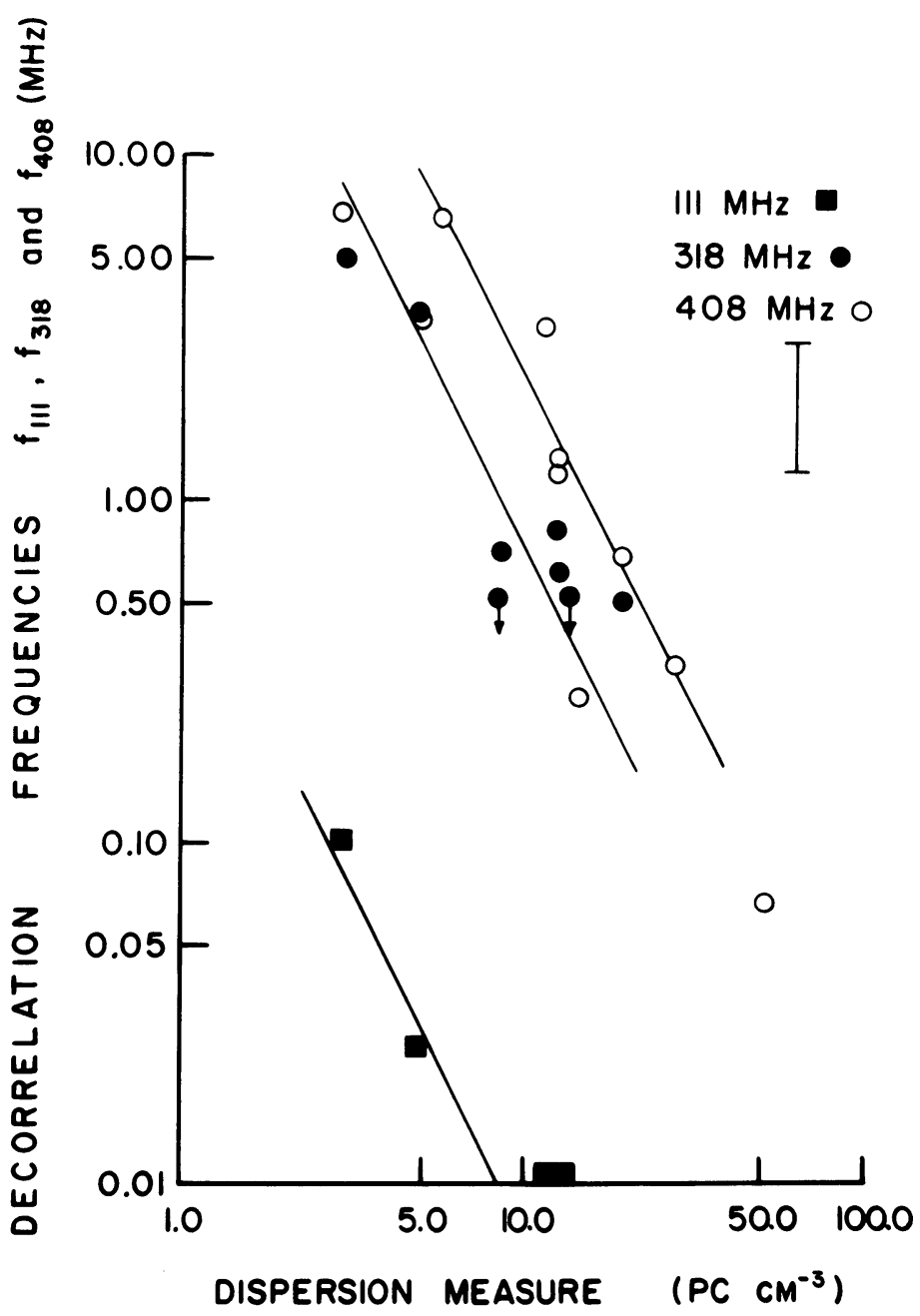

Fig. 1. Decorrelation frequencies, $f_{v}$, at $v=111,318$, and $408 \mathrm{MHz}$. The solid lines denote decorrelation frequencies which are proportional to the fourth power of observing frequency and inversely proportional to the square of the dispersion measure. The $408 \mathrm{MHz}$ data is taken from Rickett (1969) with $f_{408} \approx 0.3 B_{408}$. 
where $f_{v}$ is in $\mathrm{MHz}, v$ is the observing frequency in $\mathrm{MHz}$, and $\int n_{e} \mathrm{~d} l$ is the dispersion measure in $\mathrm{pc} \mathrm{cm}^{-3}$. Using Equations (1) and (2), the data shown in Figure 1 indicate that

$$
\theta_{\text {scat }} \approx 10^{3} \int_{D^{1 / 2} v^{2}} n_{e} \mathrm{~d} l
$$

where $\theta_{\text {scat }}$ is in seconds of arc and $D$ is in pc. Using $\int n_{e} \mathrm{~d} l \approx 56.81 \mathrm{pc} \mathrm{cm}^{-3}$ and $D \approx 2200$ pc for NP 0532 , Equation (3) indicates $\theta_{\text {scat }} \approx 0.2^{\prime \prime}$ at $80 \mathrm{MHz}$ and $1^{\prime \prime}$ at $26 \mathrm{MHz}$. These angular sizes agree well with recent measurements of the angular size of the compact source in the Crab Nebula (Bell and Hewish, 1967; Antova and Vitkevich, 1969; Cronyn, 1970a).

\section{Pulse Broadening by the Interstellar Medium}

The other major obstacle to the view that the compact source and the pulsar NP 0532 are the same object is that the compact source has not been seen to pulsate. Pulse broadening caused by interstellar scattering would, however, result in a reduction of pulsed emission and an increase in the continuum emission observed at low frequencies. Because the distribution function for the angular scattering is probably a Gaussian function, and because the scattered radiation is delayed in time relative to the unscattered radiation by $D \theta_{\text {scat }}^{2} / 4 c$, the time profile of the scattered power will be the convolution of the emitted pulse profile with an exponential function whose $1 / e$ decay time is $f_{v}^{-1}$.

It follows from Equation (2) that the pulse broadening will be proportional to $v^{-4}$, and that $f_{111}^{-1} \approx 10 \mathrm{msec}$ for the Crab pulsar. Both Staelin and Sutton (1970) and Rankin et al. (1970) have shown that $f_{111}^{-1} \approx 10 \mathrm{msec}$ for NP 0532. They also show that $f_{v}^{-1}$ is proportional to $v^{-4 \pm 1}$ for this pulsar (cf. Drake, Figure 3 ). When this scattering data is combined with flux density measurements of both the pulsar and the compact source, the data indicate that the emitted pulse spectrum is straight over the region $10 \mathrm{MHz}$ to $600 \mathrm{MHz}$, with a slope of $-2.9 \pm 0.4$ (Cronyn, 1970b and Rankin et al., 1970). Pulse broadening due to interstellar scattering accounts for the cutoff in the apparent spectrum of the pulsed radiation and for the apparent increase in the continuum flux density at low frequencies.

Although measurements of the broadening of the Crab pulses agree with extrapolations from the measurements of the $f_{v}$ of other pulsars (Figure 1), the absence of similar broadening of other pulsars caused some scientists to view the scintillation argument with skepticism. Most pulsars, however, emit wide pulses and are sufficiently close that pulse broadening due to interstellar scattering would not be detectable. In order to examine pulse broadening further, the average pulse profiles of the distant pulsar JP 1933 have been obtained (Figures 2 and 3).

The observed profiles indicate a progressive broadening at the lower frequencies which is proportional to $v^{-4}$. The $f_{111}^{-1} \approx 78 \mathrm{msec}$ for this pulsar, which has an $\int n_{e} \mathrm{~d} l$ 
of $158.6 \mathrm{pc} \mathrm{cm}^{-3}$. It is especially interesting that the $f_{111}^{-1}$ for JP 1933 is wider than that of NP 0532 by a factor which goes as the square of dispersion measure, in agreement with Equation (2). These observations show that the broadening of pulses from NP 0532 is not an isolated phenomenon, and lend additional support to the view that the compact source is the pulsar NP 0532.

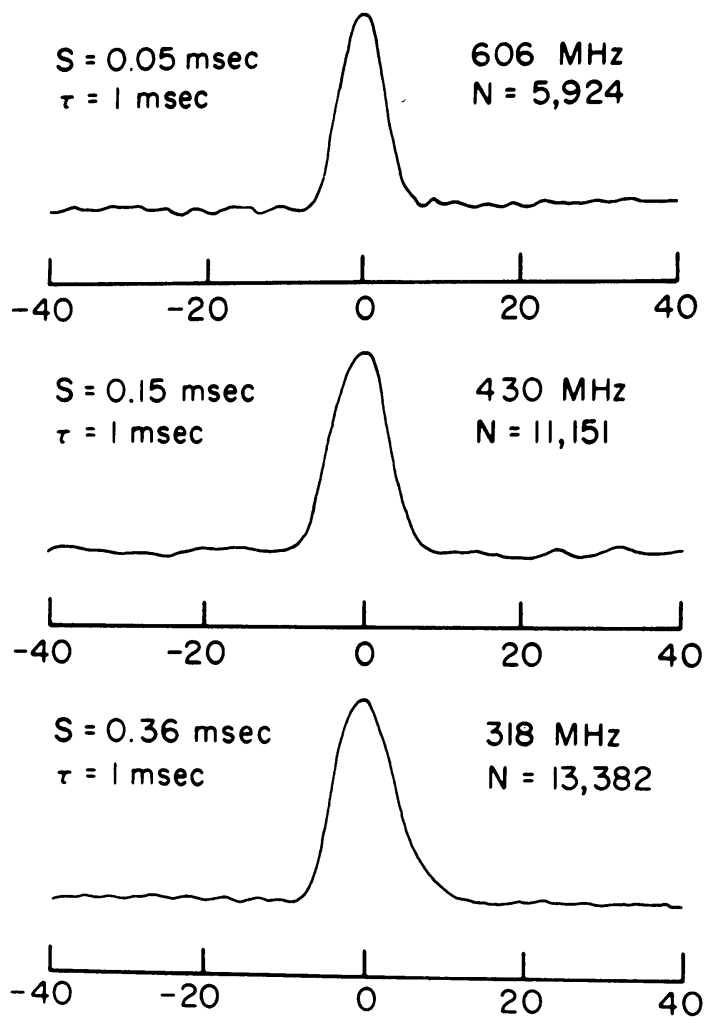

TIME IN MILLISECONDS

Fig. 2. Average pulse profiles of JP 1933 at high radio frequencies. The IF bandwidth of $10 \mathrm{kHz}$ caused a dispersion smearing of $S$, the post detection $R C$ time constant was $\tau$, and the number of pulses averaged was $N$. The zero corresponds to the zero time phase when the delay due to dispersion, $\int n_{e} \mathrm{~d} l=158.60 \pm 0.05 \mathrm{pc} \mathrm{cm}^{-3}$, is corrected for.

\section{Conclusions}

The view that the compact source is the pulsar NP 0532 is consistent with measurements of the angular size of the compact source, with measurements of the pulse broadening of NP 0532, with measurements of the spectrum of both the compact source and the pulsar, and with measurements of the interstellar scintillation parameters of many other pulsars. 


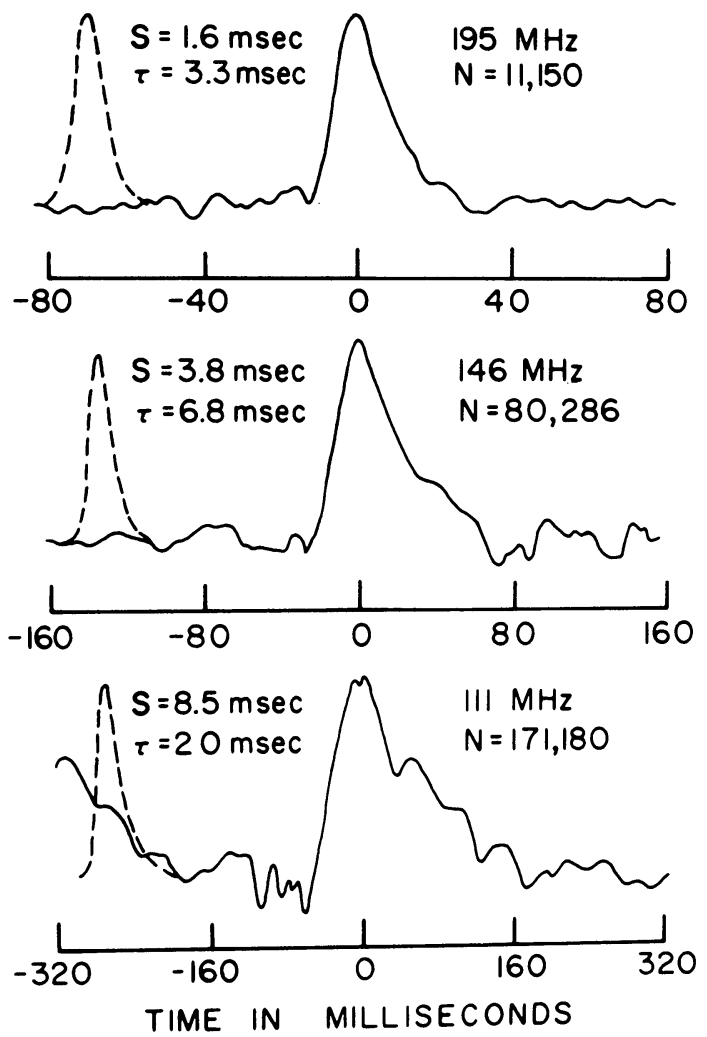

Fig. 3. Average pulse profiles of JP 1933 at low radio frequencies (solid lines). The dotted profile is that profile which would be observed if the pulse profile were independent of frequency. The IF bandwidth of $10 \mathrm{kHz}$ caused a dispersion smearing of $S$, the post detection $R C$ time constant was $\tau$, and the number of pulses averaged was $N$. The zero corresponds to the zero time phase when the delay due to dispersion, $\int n_{e} \mathrm{~d} l=158.60 \pm 0.05 \mathrm{pc} \mathrm{cm}^{-3}$, is corrected for.

\section{References}

Andrew, B. H., Branson, N. J. B. A., and Wills, D.: 1964, Nature 203, 171.

Antova, T. D. and Vitkevich, V. V.: 1969, Soviet Astron. 12, 788.

Bell, S. J. and Hewish, A.: 1967, Nature 213, 1214.

Cronyn, W. M.: 1970a, Ph.D. Thesis, Astronomy Program, University of Maryland.

Cronyn, W. M.: 1970b, Science 168, 1453.

Gower, J. F. R.: 1967, Nature 213, 1213.

Hewish, A. and Okoye, S. E.: 1964, Nature 203, 171.

Hewish, A. and Okoye, S. E.: 1965, Nature 207, 59.

Lang, K. R.: 1969, Science 166, 1401.

Lang, K. R.: 1971, Astrophys. J. 164, 249.

Rankin, J. M., Comella, J. M., Craft, H. D., Richards, D. W., Campbell, D. B., and Counselman, C. C.: 1970, Astrophys. J. 162, 707.

Rickett, B. J.: 1969, Nature 221, 158.

Staelin, D. H. and Sutton, J. M.: 1970, Nature 226, 69.

Woltjer, L.: 1968, Astrophys. J. Letters 152, L179. 
Note added in proof. W. C. Erickson, T. B. H. Kuiper, S. H. Knowles, and J. J. Broderick have recently found that the position of the pulsar NP 0532 and that of the compact source agree to $0.17^{\prime \prime}$ at $121.6 \mathrm{MHz}$. They also find the pulsating flux of the pulsar is $14 \mathrm{fu}$ whereas the unpulsating flux is $30 \mathrm{fu}$ (reported at IAU XIV General Assembly). 